\title{
Immunological Demyelination Triggers Macrophage/Microglial Cells Activation without Inducing Astrogliosis
}

\author{
Frank Cloutier, ${ }^{1,2}$ Ilse Sears-Kraxberger, ${ }^{1}$ Krista Keachie, ${ }^{1}$ and Hans S. Keirstead ${ }^{1}$ \\ ${ }^{1}$ Reeve-Irvine Research Center, Sue and Bill Gross Stem Cell Research Center, School of Medicine, University of California at Irvine, \\ 845 Health Sciences Road, Irvine, CA 92697, USA \\ ${ }^{2}$ Département de Biologie de l'Université de Moncton, 18 Antonine-Maillet, Moncton, NB, Canada E1A 3E9
}

Correspondence should be addressed to Hans S. Keirstead; hansk@uci.edu

Received 2 July 2013; Revised 16 September 2013; Accepted 24 September 2013

Academic Editor: Daniel Larocque

Copyright (C) 2013 Frank Cloutier et al. This is an open access article distributed under the Creative Commons Attribution License, which permits unrestricted use, distribution, and reproduction in any medium, provided the original work is properly cited.

\begin{abstract}
The glial scar formed by reactive astrocytes and axon growth inhibitors associated with myelin play important roles in the failure of axonal regeneration following central nervous system (CNS) injury. Our laboratory has previously demonstrated that immunological demyelination of the CNS facilitates regeneration of severed axons following spinal cord injury. In the present study, we evaluate whether immunological demyelination is accompanied with astrogliosis. We compared the astrogliosis and macrophage/microglial cell responses 7 days after either immunological demyelination or a stab injury to the dorsal funiculus. Both lesions induced a strong activated macrophage/microglial cells response which was significantly higher within regions of immunological demyelination. However, immunological demyelination regions were not accompanied by astrogliosis compared to stab injury that induced astrogliosis which extended several millimeters above and below the lesions, evidenced by astroglial hypertrophy, formation of a glial scar, and upregulation of intermediate filaments glial fibrillary acidic protein (GFAP). Moreover, a stab or a hemisection lesion directly within immunological demyelination regions did not induced astrogliosis within the immunological demyelination region. These results suggest that immunological demyelination creates a unique environment in which astrocytes do not form a glial scar and provides a unique model to understand the putative interaction between astrocytes and activated macrophage/microglial cells.
\end{abstract}

\section{Introduction}

Myelin represents a nonpermissive substrate for neuronal adhesion, sprouting, and neurite growth $[1,2]$, and several myelin-associated inhibitor proteins have been identified including the myelin-associated glycoprotein (MAG) $[3,4]$, oligodendrocyte myelin glycoprotein (OMgp) $[5,6]$ and Nogo-A [7-9]. Since then, numerous studies have been dedicated to understand the mechanisms underlying the action of these inhibitory molecules [10-12]. Previous studies in our laboratory and others have used immunological demyelination to address myelin-associated inhibition and provide a permissive environment for axonal regeneration. Immunological demyelination involves the intraspinal injection of antibodies to galactocerebroside ( $\mathrm{GalC})$, the major sphingolipid in myelin, plus complement proteins, and results in a well-defined region of complete demyelination that spares oligodendrocytes. This treatment paradigm has been shown to promote axonal regeneration following spinal cord injury in embryonic chicks [13], hatchling chicks [14], and adult rats [15-18].

One major impediment for axon regeneration following CNS injury is the formation of a glial scar $[19,20]$. This response is preceded by the transition of resident astrocytes into a reactive state rapidly following injury. Reactive astrocytes are characterized by a cellular hypertrophy and dramatic changes in gene regulation [21-24]. Notably the upregulation of GFAP has been widely used as marker of astrogliosis.

The role of reactive astrocytes in demyelinating diseases is not fully understood and both protective as well as deleterious effects are being discussed [25-27]. The presence of astrogliosis has been suggested to contribute with the failure of remyelination in many demyelinating pathologies and 
experimental models of demyelination [28-33]. In our model, remyelination begins 10 to 14 days following intraspinal injection of antibodies to GalC and remyelination of all axons is evident by 4 weeks [17]. Thus, the ability of immunological demyelination regions to sustain axonal regeneration and remyelination suggests that astrogliosis is not induced by the catastrophic destruction of myelin in this model. Such a possibility would seem paradoxical, given that astrogliosis is a ubiquitous response to different insults to the adult CNS including trauma, toxic lesion, genetic, and degenerative diseases [21, 24, 34].

In the present study we first compared the astrogliosis and macrophage/microglial cells responses 7 days after either immunological demyelination or a stab injury to the dorsal funiculus. Secondly, we compared the astrogliosis response following a stab or hemisection injury to the spinal cord dorsal funiculus within regions of immunological demyelination. Our data shows that immunological demyelination induced a robust macrophage/microglial cells activation which is not accompanied by astrogliosis either when induced alone or followed by an injury.

\section{Materials and Methods}

Adult female Sprague Dawley rats (200-220 g, 6-8 weeks old; $n=40$ ) were anaesthetized with an intraperitoneal injection of $7.5 \mathrm{mg} / \mathrm{kg}$ Rompun (Phoenix Pharmaceutical Inc., St. Joseph, MO) and $60 \mathrm{mg} / \mathrm{kg}$ Xylazine (Phoenix Pharmaceutical Inc., St. Joseph, MO). All procedures were approved by the Institutional Animal Care and Use Committee of the University of California at Irvine.

2.1. Experimental Groups. To determine whether immunological demyelination alone induced astrogliosis and activation of macrophage/microglial cells, 10 animals received an injection of GalC antibodies plus serum complement proteins into the dorsal column and were killed 7 days later for immunohistochemical $(n=6)$ or immunoelectron microscopic $(n=4)$ analyses of astroglial reactivity. To determine the extent of astrogliosis following injury, 6 animals received a stab injury in the dorsal column and 6 animals received laminectomy only and were killed 7 days later. To determine whether astrogliosis will be induced within regions of immunological demyelination accompanied with a disruption of the blood brain barrier, 12 animals received an injection of GalC antibodies plus serum complement proteins into the dorsal column, followed by a spinal cord stab wound injury to the dorsal column $(n=6)$ or dorsal spinal cord hemisection injury $(n=6) 24$ hours later, and were killed after further 7 days. 6 uninjured, normally myelinated animals served as a control group.

2.2. Immunological Demyelination. The dorsal region between the neck and hindlimbs, extending approximately $2 \mathrm{~cm}$ bilaterally from the spine, was shaved and disinfected with serial Providone iodine scrub and $70 \%$ ethanol scrubs. A midline incision exposed the spinal column at the level of T8T11 and the paravertebral muscles were dissected bilaterally to visualize the transverse processes. Laminectomy was performed at T10. After stabilization of the spinal column by clamping the T9 and T11 vertebrae, direct injection into the exposed dorsal funiculus was performed using a silicon-coated glass micropipette (with outer diameter of $50-80 \mu \mathrm{m}$ ) attached to a $10 \mu \mathrm{L}$ Hamilton syringe (Hamilton, Reno, NV) mounted on a micromanipulator. Injections consisted of polyclonal anti-GalC antibody (Chemicon International Inc., Temecula, CA) at a dilution of 1:2 with $33 \%$ guinea pig complement (ICN, Aurora, $\mathrm{OH}$ ) in artificial cerebrospinal fluid (aCSF, Harvard apparatus, Holliston, MA). Rats received a total volume of $4 \mu \mathrm{L}$ injected over 3 minutes. Muscle layers were then sutured and the superficial tissue and skin were closed with wound-clips.

2.3. Spinal Cord Injury. In animals that received immunological demyelination, the laminectomy site was reexposed 24 hours after intraspinal injection. In animals that had not previously received a demyelinating injection, laminectomy was performed as outlined above. For stab injury, a sterile 25 gauge stainless steel needle (outer diameter of $0,51 \mathrm{~mm}$ ) was inserted immediately next to the dorsal vein and lowered $1.2 \mathrm{~mm}$ into the dorsal funiculus. For hemisection injury, a microlesion knife (Fisher Scientific, Pittsburgh, PA) marked at $1.2 \mathrm{~mm}$ from the tip was lowered into the spinal cord at a depth of $1.2 \mathrm{~mm}$ from the dorsal-most point and drawn laterally across the dorsal aspect of the spinal cord. This procedure was then repeated in the opposite direction. Immediately following surgery animals received subcutaneous saline and were maintained on an isothermic pad until alert and mobile. Animals were inspected for weight loss, dehydration, and distress, with appropriate veterinary care as needed.

2.4. Histochemical Staining. Animals were sacrificed 7 days following the injection of the demyelinating agent, or 7 days following injury. Animals were terminally anaesthetized and transcardially perfused with $50 \mathrm{~mL}$ of $0.1 \mathrm{M}$ phosphate buffered saline (PBS) containing $28 \mathrm{IU} / \mathrm{mL}$ heparin (Acros, New Jersey, NY) followed by $250 \mathrm{~mL}$ of $4 \%$ paraformaldehyde (Fisher Scientific, Pittsburgh, PA) in $0.1 \mathrm{M}$ phosphate buffer (PB) $\mathrm{pH}$ 7.4. Spinal cord regions extending $1 \mathrm{~cm}$ above and $1 \mathrm{~cm}$ below the injection/injury site were postfixed in $4 \%$ paraformaldehyde in $0.1 \mathrm{M} \mathrm{PB}$ overnight then transferred to 25\% sucrose (Fisher Scientific, Pittsburgh, PA) for 24 hours. Spinal cords from stab-injured animals were cut into $1 \mathrm{~mm}$ transverse blocks and processed so as to preserve the rostral caudal sequence and orientation. Spinal cords from hemisection-injured animals were cut in the longitudinal plane. $20 \mu \mathrm{m}$ transverse or longitudinal sections were cut on a cryostat and mounted on gelatin-coated slides (Fisher Scientific, Pittsburgh, PA).

Rabbit anti-cow glial fibrillary acidic protein (GFAP) antibodies (Dako, Carpinteria, CA) were used at a dilution of $1: 2000$ in $0.5 \%$ normal goat serum (NGS) in PBS (Chemicon International Inc., Temecula, CA). Mouse anti-rat CD68 (ED-1 clone) antibodies (Serotec, Oxford, UK) were used at a dilution of $1: 200$ in $0.5 \%$ NGS in PBS. Primary antibodies were incubated overnight at $4^{\circ} \mathrm{C}$. Goat anti-rabbit or goat 
anti-mouse secondary antibodies (Alexa Fluor 488 or 594, Molecular Probes, Eugene, OR) were used at a dilution of $1: 200$ in $10 \%$ NGS in PBS. Rabbit anti-goat secondary antibodies (Alexa Fluor 546, Molecular Probes, Eugene, OR) were used at a dilution of 1:400 in 10\% rabbit serum in PBS. After 2 hours of incubation, all sections were washed in PBS and incubated for 5 minutes at room temperature in Hoechst solution (1:5000 in PBS; Molecular Probes, Eugene, OR). Standard immunohistochemical controls were included in each run. Sections were viewed and digitally photographed using an Olympus AX-80 microscope using OLYMPUS MicroSuite B3SV software (Olympus America Inc., Melville, NY).

Dorsal columns and demyelinated regions were delineated using cryostat-sectioned tissue stained with eriochrome cyanine $\mathrm{R}$ for myelin. Serial sections to those used for immunohistochemical staining were incubated at room temperature with eriochrome cyanine R (Sigma, St. Louis, MO) for 10 minutes, rinsed, and then differentiated with a $10 \%$ ferrous oxide solution (Sigma, St. Louis, MO) for 5 minutes. Morphometric analysis of dorsal column and demyelinated regions using eriochrome cyanine R-stained sections was accomplished by tracing dorsal columns and demyelinated regions within digitally photographed fields using the OLYMPUS MicroSuite B3SV software (Olympus America Inc., Melville, NY).

2.5. Electron Microscopy. Animals were transcardially perfused with $50 \mathrm{~mL}$ of $0.1 \mathrm{M}$ PBS containing $28 \mathrm{IU} / \mathrm{mL}$ heparin (Acros Inc., Geel, Belgium) followed by $250 \mathrm{~mL}$ of $4 \%$ glutaraldehyde (Fisher Scientific, Pittsburgh, PA) in $0.1 \mathrm{M}$ phosphate buffer $\mathrm{pH}$ 7.4. Spinal cord regions extending $1 \mathrm{~cm}$ above and $1 \mathrm{~cm}$ below the injection/injury site were extracted and postfixed for 24 hours in $4 \%$ glutaraldehyde. Spinal cords were cut into $1 \mathrm{~mm}$ transverse blocks and processed so as to preserve their craniocaudal sequence and orientation. The tissue blocks were rinsed in $0.1 \mathrm{M} \mathrm{PB} \mathrm{pH} 7.4$ for $30 \mathrm{~min}$, then exposed to $1 \% \mathrm{OsO}_{4}$ (Electron Microscopy Sciences, Fort Washington, PA), dehydrated in ascending alcohols, soaked in propylene oxide (Electron Microscopy Sciences, Fort Washington, PA), and embedded in Spurr resin (Electron Microscopy Sciences, Fort Washington, PA) according to standard protocols [17]. Transverse $1 \mu \mathrm{m}$ semithin sections were cut from the cranial face of each block, stained with alkaline toluidine blue to located the demyelinated regions (Sigma, St. Louis, MO), cover-slipped, and examined by light microscopy on an Olympus AX-80 microscope using OLYMPUS MicroSuite B3SV software (Olympus America Inc., Melville, NY).

2.6. Immunoelectron Microscopy. $40 \mu \mathrm{m}$ vibratome sections of glutaraldehyde-perfused tissue (above) were cut and pretreated with $10 \%$ dimethyl sulfoxide (Fisher Scientific, Pittsburgh, PA) in PBS to enhance antibody penetration, incubated in $0.1 \% \mathrm{H}_{2} \mathrm{O}_{2}$ in PBS for $10 \mathrm{~min}$, followed by incubation in PBS containing 10\% NGS. Sections were then incubated overnight at $4^{\circ} \mathrm{C}$ in rabbit anti-cow GFAP antibodies (Dako, Carpinteria, CA) at a dilution of $1: 2000$ in $0.5 \%$ NGS in PBS
(Chemicon International Inc., Temecula, CA). After several rinses with PBS the sections were incubated in biotinylated goat anti-rabbit IgG (Vector Laboratories, Burlingame, CA) at a dilution of 1:400 for 1 hour. Sections were rinsed with PBS and incubated in avidin-biotin-peroxidase complex (Vector Laboratories, Burlingame, CA) at a dilution of 1:100 for 1 hour, washed with PBS and postfixed with $2 \%$ glutaraldehyde (Fisher Scientific, Pittsburgh, PA) in PBS, rinsed with PBS and visualized with the chromogen DAB (Vector Laboratories, Burlingame, CA). Sections were postfixed in $1 \% \mathrm{OsO}_{4}$ (Electron Microscopy Sciences, Fort Washington, PA) for 1 hour, dehydrated, immersed in propylene oxide (Electron Microscopy Sciences, Fort Washington, PA), flatembedded in Spurr resin (Electron Microscopy Sciences, Fort Washington, PA) between two pieces of Aclar film (Electron Microscopy Sciences, Fort Washington, PA), and polymerized at $60^{\circ} \mathrm{C}$ for 24 hours. The embedded sections were glued onto cured resin blocks and $1 \mu \mathrm{m}$ semithin sections of the dorsal column were cut with a Leica ultramicrotome (Leica, Wetzlar, Germany), stained with toluidine blue (Sigma, St. Louis, MO) and collected on glass slides. Ultrathin sections were cut and collected on coated slotted copper grids (Electron Microscopy Sciences, Fort Washington, PA), stained with lead citrate (Sigma, St. Louis, MO) for $10 \mathrm{~min}$, and examined using a Philips CM 10 electron microscope.

2.7. GFAP and ED-1 Positive Cell Counts. To quantify activated macrophages we used the macrophage-activation antigen (ED-1), which labels a glycosylated lysosomal cytoplasmic antigen believed to be coupled with lysosomal compartments in actively phagocytic macrophages and reactive microglial cells [35-37]. To quantify astrocyte we used the GFAP marker. Transverse ED-1-stained or transverse GFAP-stained sections were viewed using an Olympus AX80 microscope (Olympus America Inc., Melville, NY). For the epicenter of injuries, for regions of distal astrogliosis or regions of immunological demyelination in the dorsal columns were located at $200 \mathrm{x}$ magnification. Images were digitally captured and a $50 \times 50 \mu \mathrm{m}\left(2500 \mu \mathrm{m}^{2}\right)$ grid was overlaid on the images using OLYMPUS MicroSuite B3SV software (Olympus America Inc., Melville, NY). Regions sampled accounted for over 10 percent of the total region of pathology for each section. For macrophages and reactive microglial cells, cells that had a clearly stained nucleus, exhibiting a rounded phagocytic morphology, and ED-1+ markers that clearly delineated the perimeter of the cell were counted. For astrocytes, cells that had a clearly stained nucleus and exhibiting GFAP markers were counted.

2.8. Semiquantitative Analysis of Distal Astrogliosis. The amount of distal astrogliosis was measured using a semiquantitative method involving computer-assisted image analysis of GFAP immunostaining (University of Texas Image Tool program V. 3). Transverse GFAP-stained sections were viewed using an Olympus AX-80 microscope (Olympus America Inc., Melville, NY). Slides contained obvious histological artifacts including tears or holes were omitted from analysis. The analysis was performed on spinal cord section $1 \mathrm{~mm}$ 
rostral to either the injury epicenter or the demyelinating agent injection site (Figure 5(a)). The dorsal columns were digitally captured at 200x magnification and a $100 \times 100 \mu \mathrm{m}$ $\left(10,000 \mu \mathrm{m}^{2}\right)$ grid was overlaid on the images using OLYMPUS MicroSuite B3SV software (Olympus America Inc., Melville, NY). Nine $10,000 \mu \mathrm{m}^{2}$ squares within the middle of the dorsal column of control animals $(n=6)$, injured animals without immunological demyelination $(n=6)$ and injured animals (stab injury) with immunological demyelination $(n=6)$, were analyzed. Within each $10,000 \mu \mathrm{m}^{2}$ region, a threshold value was assigned for positive staining by selecting genuine GFAP-positive cellular staining within the region. This method allowed us to identify only the cells and processes that stained intensely enough to fall within the threshold range first selected manually and then the same threshold range was used for all the sections. The ImageTool program then automated pixel quantification.

2.9. Statistical Analysis. Results are expressed as mean \pm SEM. The software GraphPad Prism 4.0 (L a Jolla, CA, USA) was used for all statistical comparisons. Comparison between the numbers of activated macrophage/microglial cells was analyzed using unpaired Student's $t$ tests. Data from the semiquantitative analysis of GFAP staining and the quantitative analysis of GFAP-positive cells were analyzed with a one-way ANOVA followed by a post hoc Dunnett's Multiple Comparison test. Statistical significance was set at $P<0.05$.

\section{Results}

3.1. Comparison of GFAP Immunoreactivity 7 Days following Immunological Demyelination or following a Stab Wound Injury to the Dorsal Column. In intact rats, regularly spaced, radially oriented GFAP-positive astrocytes were numerous within the dorsal white matter (Figure 1(a)). Stab injury to the dorsal column induced a classic astrocytic reaction characterized by hypertrophy and upregulation of GFAP (green) expression at 7 days after injury (Figure 1(b)). A high density of reactive astrocytes with intense GFAP expression was present within white and gray matter at the level of the injury (Figure 1(b)); the intensity of GFAP immunoreactivity at the injury site was notably increased as compared to that in the uninjured spinal cord (Figure 1(a)). Higher magnification revealed the intensely GFAP-immunoreactive white matter surrounding lesion-induced cavities bordered by a dense meshwork of reactive astrocytes with thick and long processes, which were intensely labeled with GFAP (green) (Figure 1(c)). Distal reactive astrocytes were also present $2 \mathrm{~mm}$ rostral and caudal to the injury epicenter (data not shown).

Intraspinal injection of GalC antibodies plus serum complement proteins induced a well-defined region of demyelination $\left({ }^{*}\right)$ within the dorsal column (Figure $1(\mathrm{~d})$ ). The area of immunological demyelination extended 3$4 \mathrm{~mm}$ above and $3-4 \mathrm{~mm}$ below the site of injection as previously reported $[17,18]$ (data not shown). Anti-GFAP immunostained serial sections (Figure 1(e)) indicated that immunological demyelination did not induce widespread reactive astrogliosis either within or adjacent to regions of immunological demyelination. Higher magnification showed that the edge of immunological demyelination area (hatched line) contains small non-hypertrophic astrocytes with short processes (arrows) (Figure 1(c)) which contrasted with the edge of injury bordered by a dense meshwork of reactive astrocytes (Figure 1(f)). Astrocytes were also found deeper within the regions of immunological demyelination (arrow in Figure $1(\mathrm{~g})$ ) and higher magnification showed nonhypertrophic astrocyte (arrows) and multiple GFAP positive filaments (arrow heads) (Figure $1(\mathrm{~h})$ ). This contrasted with the absence of GFAP positive cells and GFAP positive intermediate filaments within the lesion site (I) produced by the stab injury (data not shown). Quantitative analysis of GFAP-positive cells revealed no difference between the numbers of GFAP-positive cells within the normal white matter in the dorsal column compared to those within regions of immunological demyelination (Figure 1(i)). These finding confirm that astrocytes survived within regions of immunological demyelination, indicating that the lack of reactive gliosis within and surrounding regions of immunological demyelination is not due to death of astrocytes.

Further support for the survival of astrocytes and lack of astroglial reactivity within regions of immunological demyelination was obtained from electron microscopic analyses (Figure 2). GFAP immunoreactive cells were present within the regions of immunological demyelination either close to the edge (Figures 2(a)-2(b)) or deeper within the regions of immunological demyelination (Figures 2(c), 2(e), $2(\mathrm{f})$, and $2(\mathrm{~g})$ ). GFAP immunoreactivities were found within the cytoplasmic compartment immediately adjacent to the nucleus (arrows) and within processes (arrowheads). A lower magnification view (Figure 2(a)) from Figure 2(b) indicates a lack of astrogliosis within the region of immunological demyelination or at the edge (hatched line) with myelinated white matter. For comparison, distal reactive astrocyte $1 \mathrm{~mm}$ rostral to the injury epicenter without immunological demyelination possesses a hypertrophied cell body, numerous spread processes (Figure 2(d)), and thick bands of intermediate filaments within their processes (Figure 2(h)).

3.2. Macrophage/Microglial Cell Response following Spinal Cord Injury and Immunological Demyelination. The presence of activated macrophage/microglial cells within regions of immunological demyelination has been previously reported $[17,38]$. Likewise, the response of macrophage/microglial cells following hemisection, crush, or contusion injury of the rat spinal cord has been thoroughly described [3942] and found to be maximal between 3 and 7 days after injury. In the present study, we quantitatively compared macrophage/microglial cell response 7 days following immunological demyelination or stab injury to the dorsal column. Eriochrome cyanine R staining revealed welldefined regions of immunological demyelination within the center of the dorsal column (Figure 3(a)). Double anti-ED-1 (red) and GFAP (green) immunostained serial sections to A (Figure 3(b)) revealed that immunological 


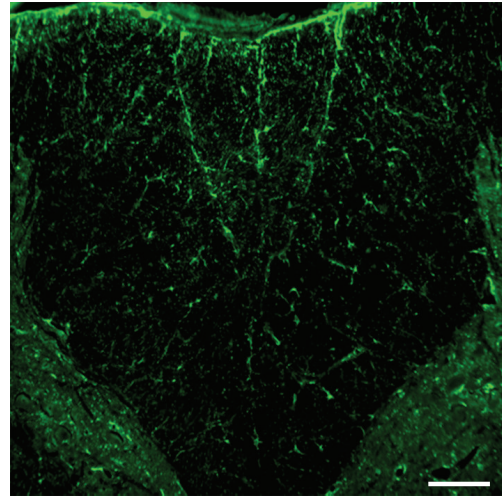

(a)

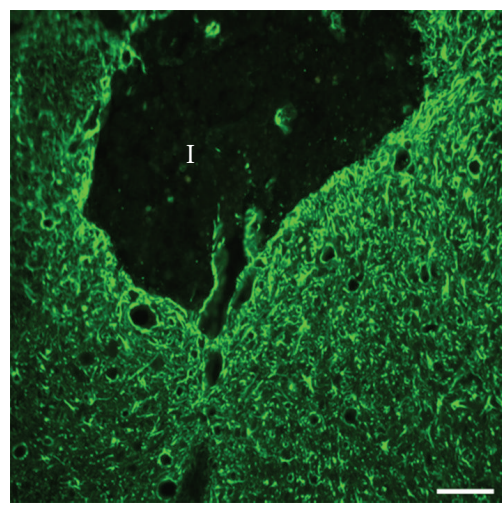

(b)

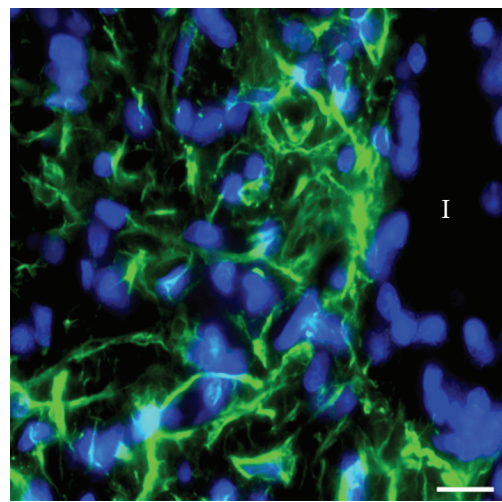

(c)

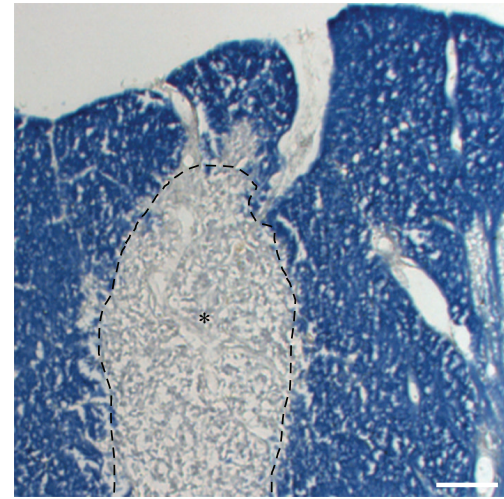

(d)

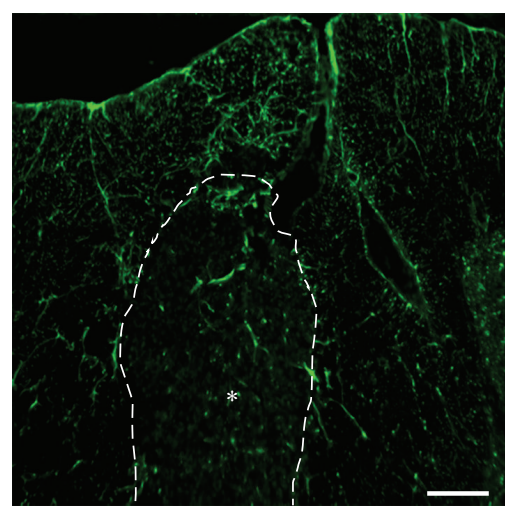

(e)

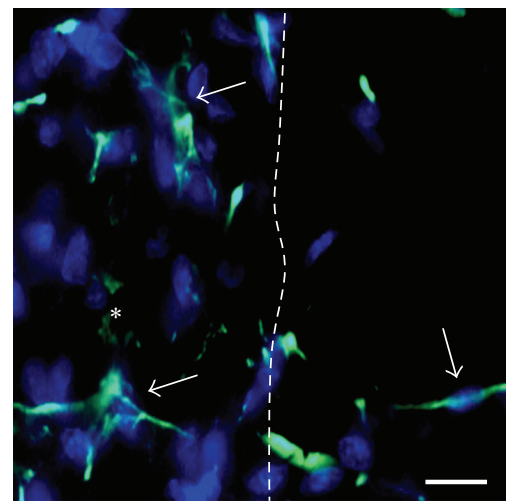

(f)

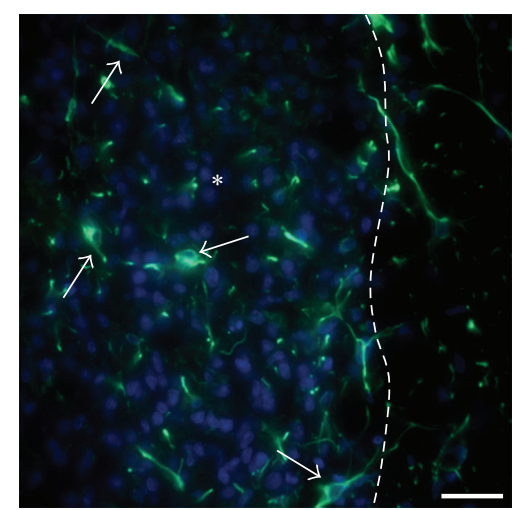

(g)

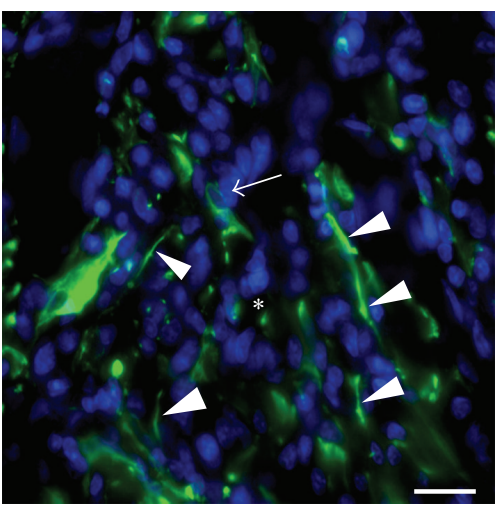

(h)

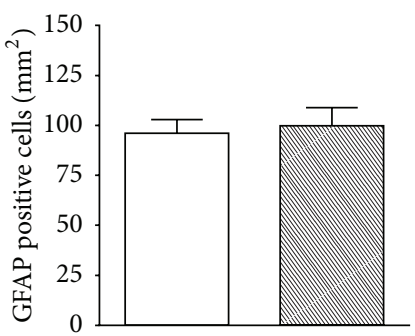

$\square$ Within spinal cord dorsal column

$\square$ Within the regions of immunological demyelination

(i)

FIGURE 1: Comparison of the expression of GFAP 7 days after a spinal cord stab injury to the dorsal column or after an injection of GalC antibodies plus complement proteins to the dorsal column. (a) The intensity of GFAP (green) immunoreactivity is low in the uninjured spinal cord dorsal column. (b) A dense ring of reactive astrocytes with intense GFAP (green) expression is evident surrounding the lesion "I." (c) Higher magnification of the lesion edge: the double staining for GFAP and Hoechst reveals many reactive astrocytes tightly juxtaposed each other. (d) Eriochrome cyanine R staining showing the region of immunological demyelination. (e) GFAP (green) staining of a serial section to (d). (f) Higher magnification view within the region of demyelination showing double staining of GFAP (green) and Hoechst (blue). Note the presence of GFAP positive astrocytes (arrows) close to the edge of immunological demyelination region (white ${ }^{*}$ ). (g) GFAP positive astrocytes (arrows) deeper within the region of demyelination. Note the absence of reactive astrocytes at the border zone between normally myelinated and demyelinated area. (h) Higher magnification within the region of demyelination: the double staining for GFAP and Hoechst reveals an astrocyte (arrow) and multiple intermediate GFAP filaments (arrow heads). (i) The numbers of GFAP-positive cells within regions of immunological demyelination were similar compared to those within regions of normal dorsal column. Scale bars: (a) and (b) $100 \mu \mathrm{m}$; (d), (e), and (g) $50 \mu \mathrm{m}$; (c), (f), and (h) $16.6 \mu \mathrm{m}$. 


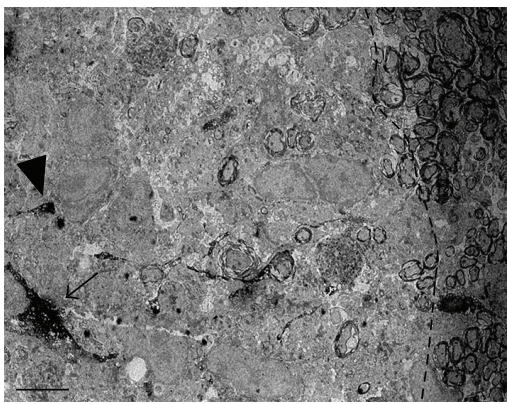

(a)

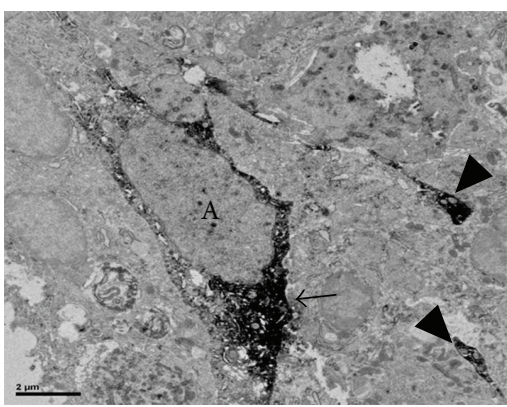

(b)

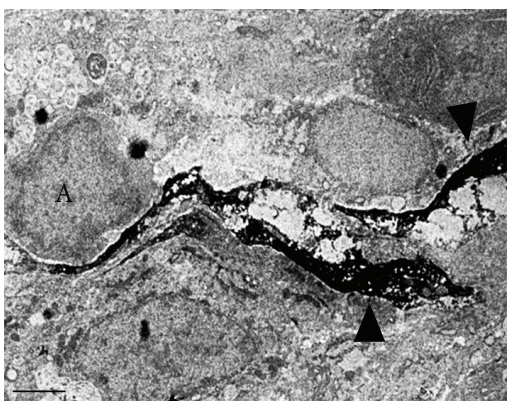

(c)

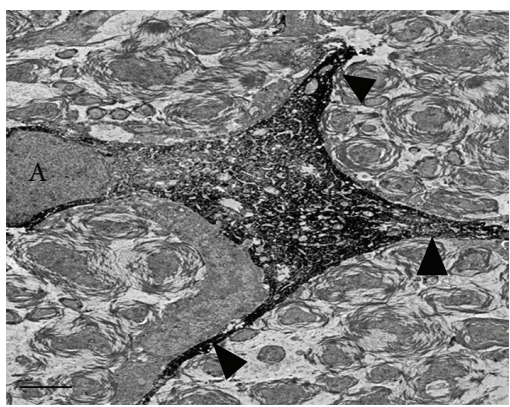

(d)

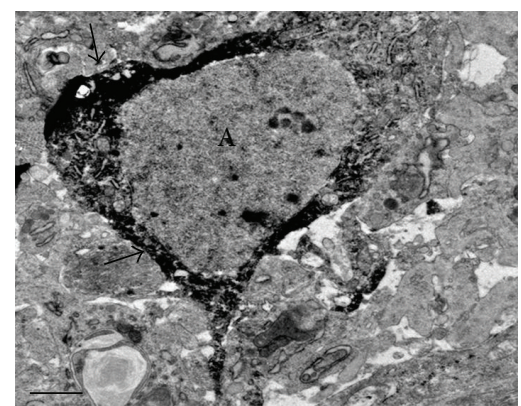

(e)

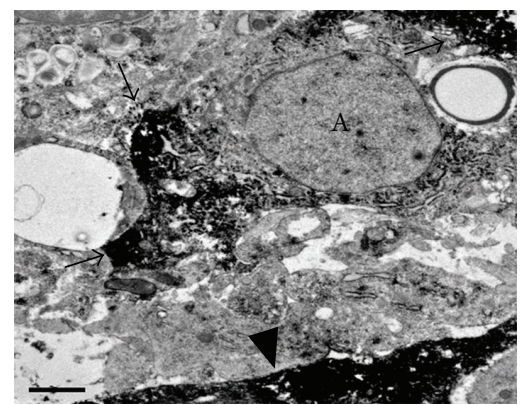

(f)

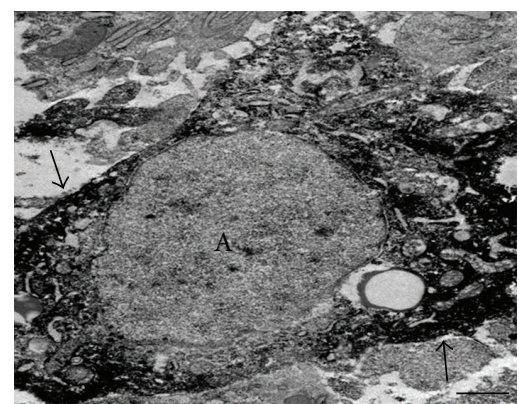

(g)

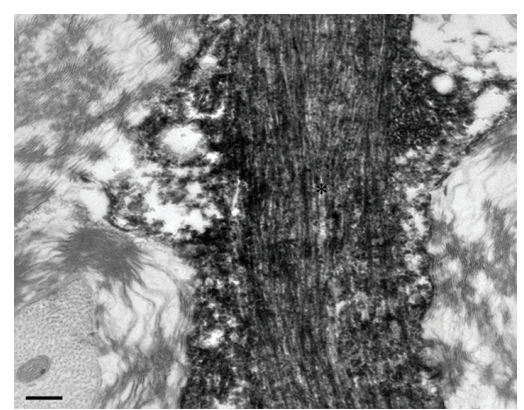

(h)

FIGURE 2: Localization of GFAP-immunoreactivity by immunoelectron microscopy within the region of immunological demyelination 7 days following intraspinal injection of GalC antibodies plus complement proteins. (a) Lower magnification of (b) showing a GFAP-positive astrocyte and the nearby border zone between the normally myelinated and demyelinated region (hatched line). Note the lack of astrogliosis at the border zone. (b)-(g) Astrocyte (A) with GFAP-immunoreactivity (arrows) within the cytoplasmic compartment immediately adjacent to the nucleus and a GFAP-positive process (arrowhead). For comparison, (d) illustrates distal reactive astrocytes $1 \mathrm{~mm}$ rostral to the injury epicenter. Note the hypertrophied process and multiple branches (arrowheads). (h) High magnification with focus on GFAP-positive process (black ${ }^{*}$ ). Note multiple dark GFAP-positive parallel lines corresponding to intermediate filaments. Scale bars: (a) $5 \mu \mathrm{m}$; (b), (c), and (f) $2 \mu \mathrm{m}$; (d) $5 \mu \mathrm{m}$; (e) and (g) $1 \mu \mathrm{m}$; (h) $0,2 \mu \mathrm{m}$. 


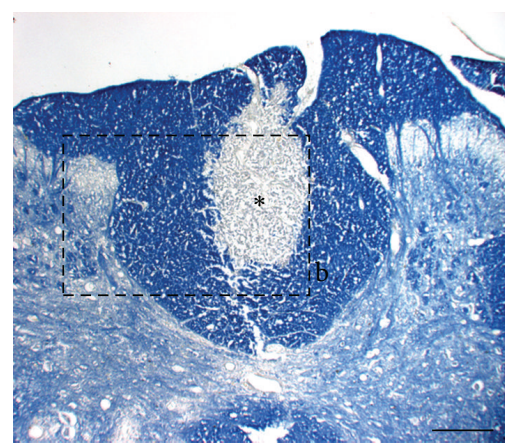

(a)

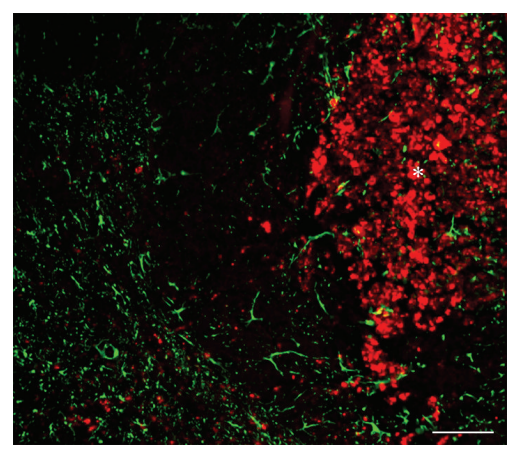

(b)

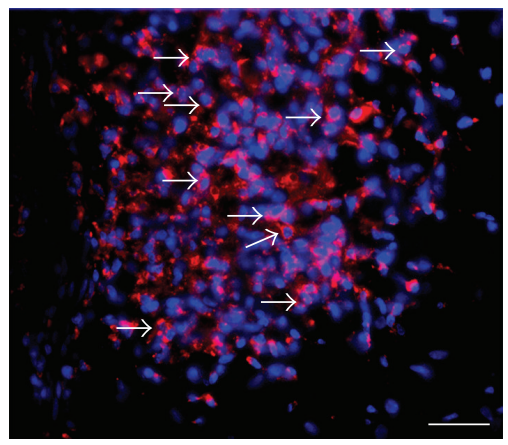

(c)

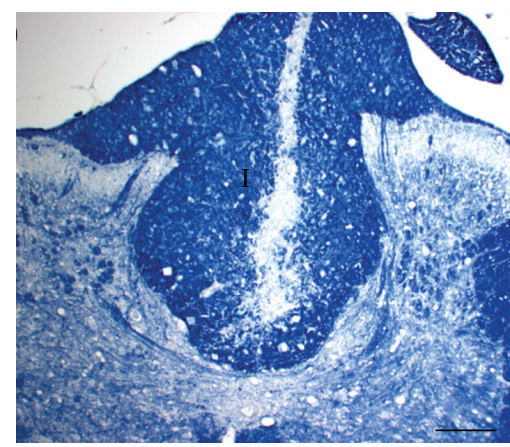

(d)

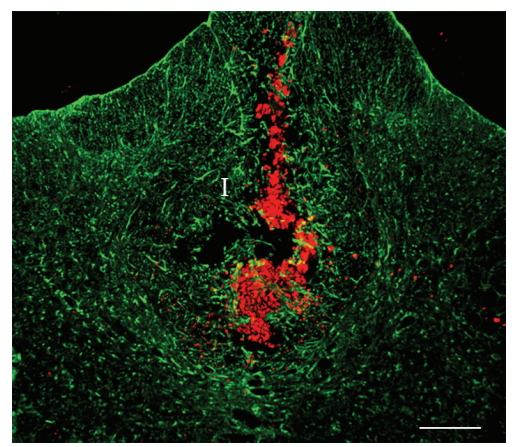

(e)

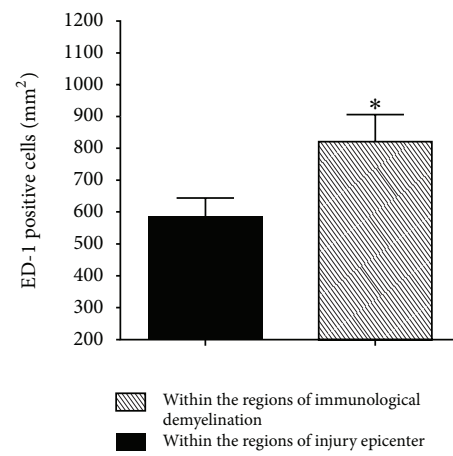

(f)

FIGURE 3: Macrophage-activation antigens (ED-1) 7 days following intraspinal injection of GalC antibodies plus complement proteins or a spinal cord stab injury to the dorsal column. (a) Eriochrome cyanine R-stained transverse section showing the region of immunological demyelination $\left(^{*}\right)$. (b) Double stained GFAP (green) and ED-1 (red) serial section to (a). The inset highlights the numerous ED-1 positive cells within the region of immunological demyelination (white ${ }^{*}$ ) and the absence of astrogliosis. (c) Localization of ED-1 positive cells (arrows) within the region of immunological demyelination (hatched line). Activated macrophages characterized by their large round cell bodies and their high immunoreactivity for ED-1 are distributed homogeneously throughout the whole region of immunological demyelination. (d) Eriochrome cyanine R-stained transverse section showing the injury site (I). (e) Double stained GFAP (green) and ED-1 (red) serial section to (d). Numerous ED-1 positive cells are present within the injury site bordered by numerous GFAP positive-hypertrophic astrocytes. (f) Quantitative comparisons between the number of ED-1+ cells per $\mathrm{mm}^{2}$ found within injury epicenters and within regions of immunological demyelination $\left(^{*}\right), P<0.05$. Scale bars: (a), (d), and (e) $200 \mu \mathrm{m}$; (b) and (c) $50 \mu \mathrm{m}$.

demyelination is accompanied by a robust activation of macrophage/microglial cells without astrogliosis response. Numerous activated macrophages characterized by their large round cell bodies and their high immunoreactivity for ED-1 were distributed homogeneously throughout the whole region of immunological demyelination (arrows, Figure 3(c)). Notably, macrophage/microglial cells were restricted almost exclusively to regions of immunological demyelination, with only a few cells outside of the region of immunological demyelination (Figure 3(c)) or rostral and caudal to the region of immunological demyelination (data not shown). Supporting our previous observations in this study, the region of immunological demyelination was accompanied by astrogliosis (Figure 3(b)).

For the stab injury to the dorsal funiculus, the trace of the needle tract and accompanying damage to the dorsal column were revealed by the eriochrome cyanine $\mathrm{R}$ staining (Figure 3(d)). ED-1+ cells were found mainly at the injury 


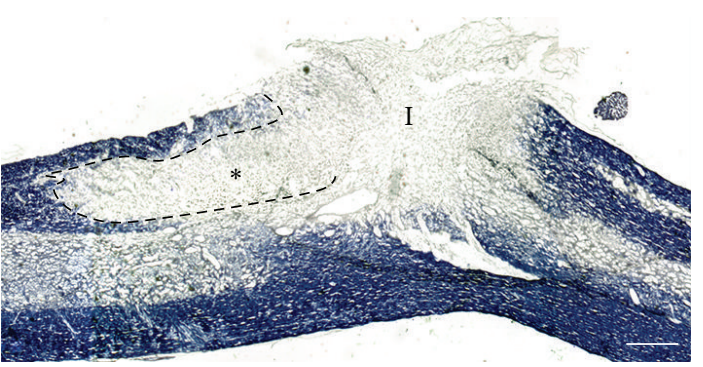

(a)
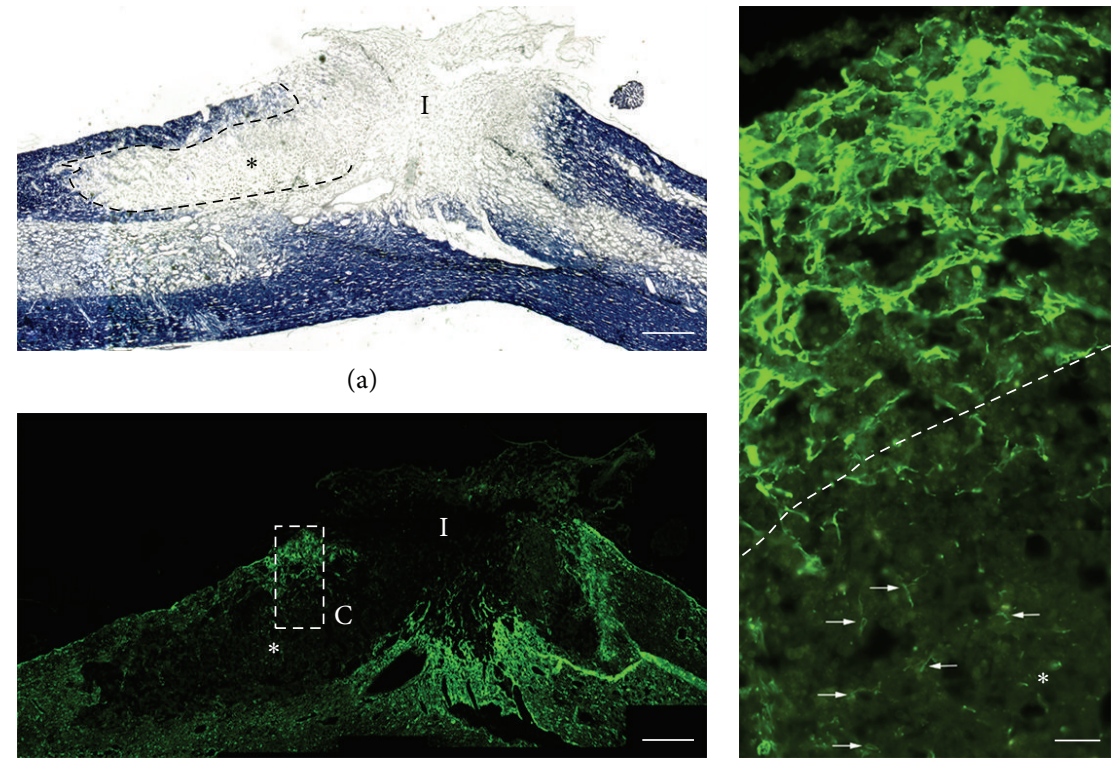

(b)

(c)

FIGURE 4: Immunological demyelination prevents astroglial hypertrophy following dorsal hemisection injury. (a) Eriochrome cyanine Rstained parasagittal section showing the extent of the demyelination $\left(^{*}\right)$ in the dorsal column rostral to the hemisection injury site (I). (b) GFAP-stained parasagittal serial section to (a). Astroglial hypertrophy is evident surrounding the hemisection injury, but is absent within the region of immunological demyelination $\left(^{*}\right)$. (c) Higher magnification of box in (b) showing the edge of the region of gliosis within normal white matter above the region of demyelination (hatched line indicates the border of the region of demyelination). The arrows demark a few of the many astrocytes present within the region of demyelination. Note that these astrocytes are small and have short and thin processes characteristic of quiescent astrocytes, which contrast with the hypertrophic astrocytes within the region of gliosis immediately above. Scale bars: (a) and (b) $400 \mu \mathrm{m}$; (c) $50 \mu \mathrm{m}$.

epicenter in all cases (Figure 3(e)). Few ED-1 positive cells were scattered throughout peripheral zones of ventral and lateral white matter rostral and caudal to the epicenter (data not shown). Quantitative analysis showed a statistically significant difference between the number of ED-1+ cells per $\mathrm{mm}^{2}$ found within the injury epicenters and within regions of immunological demyelination without injury $\left({ }^{*}\right)$ $P<0.05$ (Figure 3(f)). These findings indicate that both immunological demyelination and stab injury induce a strong macrophage/microglial cell response despite differing astrogliotic responses.

3.3. Combination of Immunological Demyelination and Spinal Cord Injury. Previous studies by Silver group and others have observed a correlation between the most significant blood brain barrier (BBB) breakdown and the greatest astrogliosis formation [43-46]. To determine whether a breakdown of $\mathrm{BBB}$ within the regions of immunological demyelination induced astrogliosis, we first created a region of immunological demyelination in the dorsal column and then $24 \mathrm{~h}$ later an hemisection or a stab injury was performed.

Findings from hemisection are presented in Figure 4. In hemisection-injured animals, eriochrome cyanine Rstained midline longitudinal sections clearly revealed the dorsal and ventral white matter tracks (blue stain) within the spinal cord (Figure 4(a)). Regions of immunological demyelination appeared as white bands within the dorsal column and extended several millimeters rostral and caudal to the hemisection injury site (labeled I in Figure 4(a)). Serial sections stained for GFAP revealed robust astroglial hypertrophy and GFAP expression around the injury site but not within regions of immunological demyelination; astrocytes within regions of immunological demyelination did not display the intense GFAP staining typical of reactive astrocytes either at a distance from or immediately adjacent to the base of the lesion (Figure 4(b)). Higher magnification of the white matter adjacent to the injury site confirmed robust astroglial hypertrophy and GFAP expression within normally myelinated white matter, but a lack of astroglial reactivity within regions of immunological demyelination; GFAP immunostaining within regions of immunological demyelination revealed astrocytes with short, thin processes characteristic of quiescent cells (arrows in Figure 4(c)). The number and distribution of astrocytes within regions of immunological demyelination appeared similar to the number and distribution of astrocytes within normal white matter.

Quantitative analysis of GFAP-positive cells and semiquantitative analysis of GFAP staining are depicted in Figure 5. Schematic drawing shows where the analyses were conducted (Figure 5(a)). Since it was difficult to distinguish regions of immunological demyelination at the injury epicenters due to the destruction and distortion of tissues, these analyses were conducted $1 \mathrm{~mm}$ rostral to the injury epicenters (stab injury) (Figures 5(b)-5(c)) where astrogliosis was still predominant and clear delineated regions of immunological demyelination with eriochrome cyanine $\mathrm{R}$ staining could be identified (Figures 5(d)-5(g)). In animals that received a 


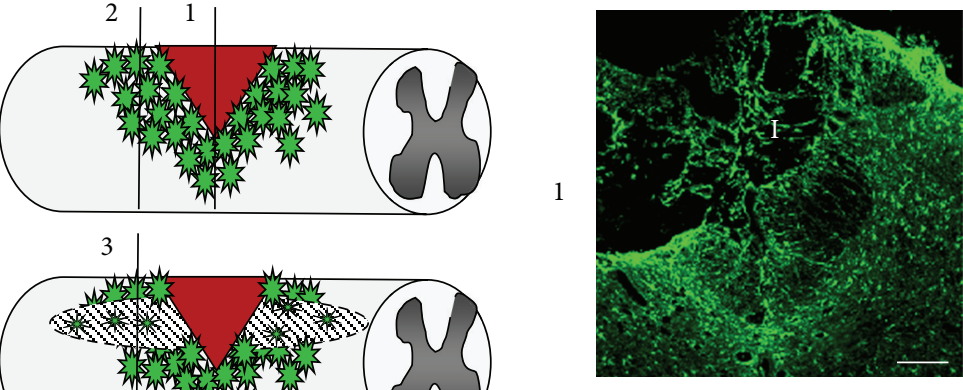

(b)

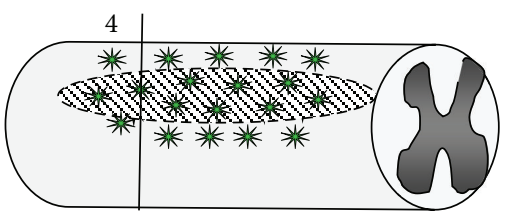

* Normal astrocyte

Reactive astrocyte

artos Immunological demyelination

Injury site

(a)

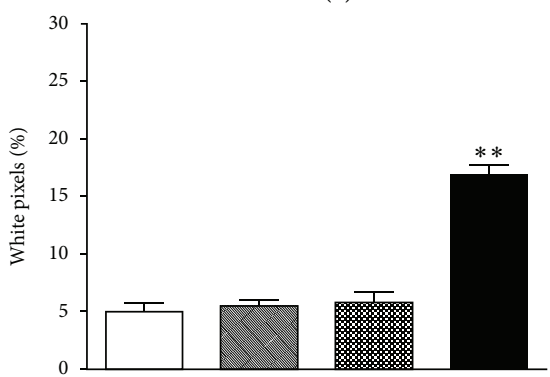

$\square$ Control group

Within the regions of immunological demyelination (a4)

Within the regions of immunological demyelination (a3)

- Within the regions of distal astrogliosis (a2)

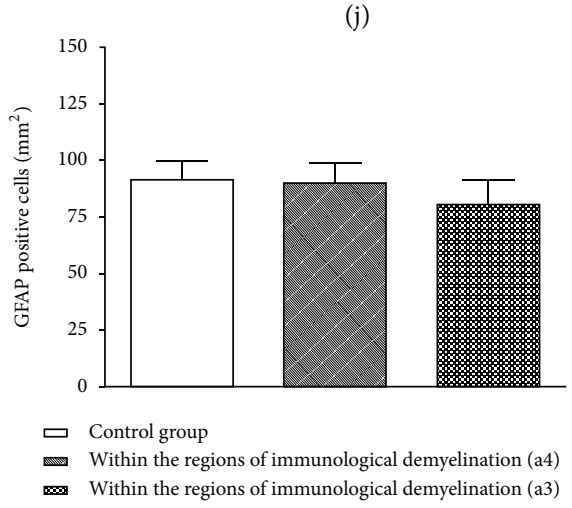

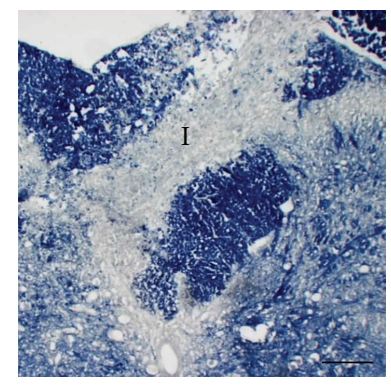

(c)

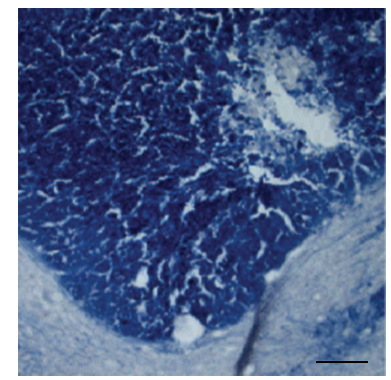

(e)

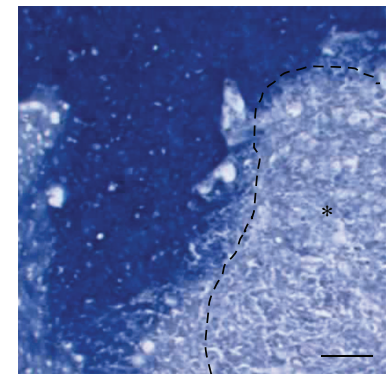

(g)

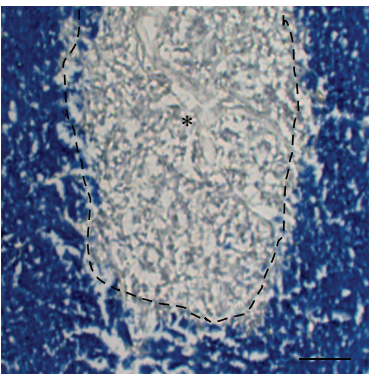

(i)

(k)

FIGURE 5: Immunological demyelination attenuates the induction of distal reactive. (a) Schematic drawing of different experimental groups and where analysis of white pixel and GFAP-positive cell counts were conducted. (b) GFAP-stained serial section to (c). The injury site is surrounded by wide spread astrogliosis in the gray and the white matter. (c) Eriochrome cyanine R-stained transverse section showing the injury site (I). (d) GFAP-stained serial section to (e). Astrogliosis is still evident $1 \mathrm{~mm}$ rostral the injury epicenter. (f) GFAP-stained serial section to (g). (f) Astrogliosis is markedly reduced within the region of demyelination ( ${ }^{*}$, hatched line). (e) Eriochrome cyanine R-stained transverse section showing the region of demyelination $1 \mathrm{~mm}$ rostral the injury epicenter. (j) Whereas there is a significant increase in $\%$ of GFAP staining $1 \mathrm{~mm}$ rostral to the injury epicenter, regions of immunological demyelination showed a similar percentage of GFAP staining compared to normal white matter. (k) The numbers of GFAP-positive cells within regions of immunological demyelination either alone or $1 \mathrm{~mm}$ rostral to the injury epicenter are similar compared to those of the normal dorsal column. ${ }^{* *}: P<0.01$. Scale bars: (b)-(c) $100 \mu \mathrm{m}$; (d) $-(i) 50 \mu \mathrm{m}$. 
stab injury without previous immunological demyelination, GFAP stained transverse sections revealed a dense population of distal hypertrophic reactive astrocytes intensely expressing GFAP in the dorsal column $1 \mathrm{~mm}$ rostral (Figure 5(d)) to the injury epicenter (Figure 5(b)). However, in animals that underwent immunological demyelination, hypertrophic astrocytes were absent within the demyelinated area $\left({ }^{*}\right)$ but present in surrounding tissue (Figure 5(f)). GFAP pixel quantification revealed a statistically significant decrease within regions of immunological demyelination (hatched line) compared to regions of astrogliosis without immunological demyelination $1 \mathrm{~mm}$ rostral to the injury epicenter; $16.74 \pm 1.18 \%$ to $5.59 \pm 0.82 \%, n=6, P<0.01$ (Figure $5(\mathrm{j})$ ). No statistical difference was found between the pixel content within regions of immunological demyelination and within the dorsal column of control animals (Figure 5(j)).

\section{Discussion}

In this study we report that immunological demyelination is not accompanied by astrogliosis despite causing intense macrophage/microglial activation and document a novel animal model to understand the putative physiological response of astrocytes during inflammation in the central nervous system.

\subsection{Survival of Astrocyte within Immunological Demyeli-} nation. The lack of astroglial reactivity within regions of immunological demyelination raises the question whether this treatment might just kill astrocytes. For instance, other models of demyelination, such as intraspinal injection of ethidium bromide, create a myelin-free region in which astrocytes die and consequently no astroglial reactivity is seen within the myelin-free region [47-49]. In the present study, immunohistochemical and immunoelectron microscopic assays revealed GFAP positive cells within regions of immunological demyelination, indicating that astrocytes survived. Furthermore, our quantitative analysis indicated that regions of immunological demyelination contained a number of GFAP-positive cells that were not statistically different compared to normal dorsal white matter. Similarly, one previous study using intraspinal injection of anti-GalC observed a survival of astrocytes but a reduction of GFAP staining, although no quantitative data was presented [50]. It is noteworthy that immunological demyelination is followed by complete remyelination by oligodendrocytes [17] and numerous studies showed that astrocytes are needed for oligodendrocyte remyelination [51-55]. Although we showed that immunological demyelination with anti-GalC does not kill astrocytes, further investigation will be needed to clarify whether regions of immunological contains newly formed astrocytes or dying astrocytes.

4.2. Why Immunological Demyelination Is Not Accompanied by Astrogliosis? Increased expression of GFAP is a hallmark of astrogliosis and our semiquantitative analysis indicated that regions of immunological demyelination contained a GFAP density that was not statistically different from the
GFAP density in normal spinal cords. Astrocytes are distributed throughout the whole CNS and become reactive in response to essentially any disturbance, including trauma, stroke, neurotoxic damage, genetic, and neurodegenerative diseases, for reviews [21, 24, 34]. Regarding animal models of demyelination, an increase in astrogliosis has been previously reported during cuprizone-induced demyelination [56-58] as well as with the lysolecithin demyelination model [5961]. Immunological demyelination itself represents as well an insult to the CNS. Immunological demyelination involves the targeting of antibodies against the major sphingolipid in myelin (galactocerebroside) and the subsequent activation of the complement cascade, leading to the formation of membrane attack complexes and myelin destruction within hours of administration [13, 38, 62].

Many different types of molecules that can be generated through a wide variety of different mechanisms are able to trigger features of reactive astrogliosis. Molecular mediators of reactive astrogliosis can be released by any cell type in central nervous system tissue, including neurons, microglia, oligodendrocyte lineage cells, endothelia, leukocytes, and astrocytes, reviewed in [23, 24]. Immunological demyelination is accompanied by a large population of activated macrophage/microglial cells. Activated macrophage/microglial cells can release putative triggers of astrogliosis [22] like IL-1 $\beta$ [63-68], IL-6 [69-71] and TNF$\alpha$ [67]. Conversely, the anti-inflammatory cytokine interleukin (IL)-10, a potent inhibitor of cytokine synthesis from microglia and macrophages [72-76], has been reported to reduce glial scaring [77]. Other evidence for a role of inflammatory mononuclear cell cytokines as mediators of astrogliosis is coming from studies on CNS development. Indeed, one hypothesis to explain the lack of astrogliosis during CNS development is the relative immaturity of the immune system in neonates compared to adults [78-81]. Yong and coworkers showed that the inability of neonatal astrocytes to become reactive can be reversed by microinjection of gamma-IFN, IL-1, IL-2, IL-6, TNF- $\alpha$, and macrophage colony stimulating factor (M-CSF), which resulted in a significant increase of astrogliosis in neonatal mouse brain, similar to that seen in an adult [82]. In the cuprizone-induced demyelination model, astrogliosis promptly followed macrophage/microglia activation [57] and an increased expression of many inflammatory cytokines including TNF- $\alpha$ and IL- $1 \beta$ has been demonstrated within regions of demyelination. Using the same model, Skripuletz and colleagues demonstrated that reactive astrocytes provide the signal environment that forms the bases for the recruitment of microglia to clear myelin debris [58]. With the lysolecithin demyelination model, many cytokines triggers of astrogliosis including TNF- $\alpha$ and IL$1 \beta$ are release as well $[61,83,84]$. Therefore the presence of a large population of activated macrophage/microglial cells within regions of immunological demyelination and the concomitant lack of astrogliosis is quite unique. Clearly a different signaling occurs within regions of immunological demyelination which does not encourage astrocytes to become hypertrophic and form a glial scar.

The lack of blood brain barrier (BBB) breakdown represents another hypothesis. Previous studies by Silver group 
and others have observed a correlation between the most significant BBB breakdown and the greatest scar formation [43-46]. These observations lead to the hypothesis that extravasation of serum components might trigger astrogliosis. Supporting this view, Schachtrup et al. [85] demonstrated that the blood protein fibrinogen, which leaks into the CNS immediately after BBB disruption or vascular damage, serves as an early signal for the induction of glial scar formation via the TGF- $\beta /$ Smad signaling pathway $[85,86]$. It is noteworthy that we observed reactive astrocytes around the needle insertion site for the injection of anti-galactocerebroside antibodies and complement proteins serum suggests that the lack of astrogliosis might correlate with the lack of BBB breakdown. However, this hypothesis does not explain why distal astrocytes do not become reactive with hypertrophied bodies within regions of immunological demyelination overlapping the extended area of distal astrogliosis several millimeters above or below the stab wound injury site. It is likely that distal astrocytes caudal or rostral to the lesion are exposed to all the astrogliosis mediators released following the BBB breakdown and should become reactive and hypertrophied. Again, this observation raises the hypothesis that a different signaling occurs within regions of immunological demyelination compared to other experimental models of demyelination.

In conclusion, our results indicate that immunological demyelination triggers a robust activated macrophage/ microglial cells response without inducing astrogliosis. Nonetheless, several questions remain to be addressed. How does immunological demyelination affect astrocyte metabolism and expression of other markers of reactive astrocyte? Has immunological demyelination simply slowed the astrocyte response, killed a percentage of astrocytes, or even metabolically damaged them so as to decrease their ability to generate GFAP? Immunological demyelination offers a unique model to understand the putative physiological response of astrocytes and their molecular interplay with activated macrophage/microglial cells during inflammation in the central nervous system.

\section{Acknowledgments}

The authors thank Gabriel Nistor for general advice and assistance with imaging and Adriana Gutierrez, Emma Flores, Charlie Mendoza, Julio Espinosa, and Vadim Fedulov for assistance with animal care. This project was supported by NIH (R-01-NS-41484). Hans. S. Keirstead is Chairman of the Scientific Advisory Board of and has financial interest in California Stem Cell.

\section{References}

[1] P. Caroni and M. E. Schwab, "Antibody against myelin associated inhibitor of neurite growth neutralizes nonpermissive substrate properties of CNS white matter," Neuron, vol. 1, no. 1, pp. 85-96, 1988.

[2] P. Caroni and M. E. Schwab, "Two membrane protein fractions from rat central myelin with inhibitory properties for neurite growth and fibroblast spreading," Journal of Cell Biology, vol. 106, no. 4, pp. 1281-1288, 1988.

[3] L. McKerracher, S. David, D. L. Jackson, V. Kottis, R. J. Dunn, and P. E. Braun, "Identification of myelin-associated glycoprotein as a major myelin-derived inhibitor of neurite growth," Neuron, vol. 13, no. 4, pp. 805-811, 1994.

[4] G. Mukhopadhyay, P. Doherty, F. S. Walsh, P. R. Crocker, and M. T. Filbin, "A novel role for myelin-associated glycoprotein as an inhibitor of axonal regeneration," Neuron, vol. 13, no. 3, pp. 757-767, 1994.

[5] V. Kottis, P. Thibault, D. Mikol et al., "Oligodendrocyte-myelin glycoprotein (OMgp) is an inhibitor of neurite outgrowth," Journal of Neurochemistry, vol. 82, no. 6, pp. 1566-1569, 2002.

[6] X. Wang, S.-J. Chun, H. Treloar, T. Vartanian, C. A. Greer, and S. M. Strittmatter, "Localization of Nogo-A and Nogo-66 receptor proteins at sites of axon-myelin and synaptic contact," Journal of Neuroscience, vol. 22, no. 13, pp. 5505-5515, 2002.

[7] M. S. Chen, A. B. Huber, M. E. Van Der Haar et al., "Nogo-A is a myelin-associated neurite outgrowth inhibitor and an antigen for monoclonal antibody IN-1," Nature, vol. 403, no. 6768, pp. 434-439, 2000.

[8] T. GrandPré, L. I. Shuxin, and S. M. Strittmatter, "Nogo66 receptor antagonist peptide promotes axonal regeneration," Nature, vol. 417, no. 6888, pp. 547-551, 2002.

[9] R. Prinjha, S. E. Moore, M. Vinson et al., "Inhibitor of neurite outgrowth in humans," Nature, vol. 403, no. 6768, pp. 383-384, 2000.

[10] F. Akbik, W. B. J. Cafferty, and S. M. Strittmatter, "Myelin associated inhibitors: a link between injury-induced and experiencedependent plasticity," Experimental Neurology, vol. 235, no. 1, pp. 43-52, 2012.

[11] J. W. Fawcett, M. E. Schwab, L. Montani, N. Brazda, and H. W. Muller, "Defeating inhibition of regeneration by scar and myelin components," Handbook of Clinical Neurology, vol. 109, pp. 503$522,2012$.

[12] J. K. Lee and B. Zheng, "Role of myelin-associated inhibitors in axonal repair after spinal cord injury," Experimental Neurology, vol. 235, no. 1, pp. 33-42, 2012.

[13] H. S. Keirstead, S. J. Hasan, G. D. Muir, and J. D. Steeves, "Suppression of the onset of myelination extends the permissive period for the functional repair of embryonic spinal cord," Proceedings of the National Academy of Sciences of the United States of America, vol. 89, no. 24, pp. 11664-11668, 1992.

[14] H. S. Keirstead, J. K. Dyer, G. N. Sholomenko, J. McGraw, K. R. Delaney, and J. D. Steeves, "Axonal regeneration and physiological activity following transection and immunological disruption of myelin within the hatchling chick spinal cord," Journal of Neuroscience, vol. 15, no. 10, pp. 6963-6974, 1995.

[15] R. Azanchi, G. Bernal, R. Gupta, and H. S. Keirstead, "Combined demyelination plus Schwann cell transplantation therapy increases spread of cells and axonal regeneration following contusion injury," Journal of Neurotrauma, vol. 21, no. 6, pp. 775-788, 2004.

[16] J. K. Dyer, J. A. Bourque, and J. D. Steeves, "Regeneration of brainstem-spinal axons after lesion and immunological disruption of myelin in adult rat," Experimental Neurology, vol. 154, no. 1, pp. 12-22, 1998.

[17] H. S. Keirstead and W. F. Blakemore, "Identification of postmitotic oligodendrocytes incapable of remyelination within the demyelinated adult spinal cord," Journal of Neuropathology and Experimental Neurology, vol. 56, no. 11, pp. 1191-1201, 1997. 
[18] H. S. Keirstead, H. C. Hughes, and W. F. Blakemore, "A quantifiable model of axonal regeneration in the demyelinated adult rat spinal cord," Experimental Neurology, vol. 151, no. 2, pp. 303-313, 1998.

[19] J. W. Fawcett and R. A. Asher, "The glial scar and central nervous system repair," Brain Research Bulletin, vol. 49, no. 6, pp. 377391, 1999.

[20] J. Silver and J. H. Miller, "Regeneration beyond the glial scar," Nature Reviews Neuroscience, vol. 5, no. 2, pp. 146-156, 2004.

[21] M. Eddelston and L. Mucke, "Molecular profile of reactive astrocytes-implications for their role in neurologic disease," Neuroscience, vol. 54, no. 1, pp. 15-36, 1993.

[22] J. L. Ridet, S. K. Malhotra, A. Privat, and F. H. Gage, "Reactive astrocytes: cellular and molecular cues to biological function," Trends in Neurosciences, vol. 20, no. 12, pp. 570-577, 1997.

[23] M. V. Sofroniew, "Molecular dissection of reactive astrogliosis and glial scar formation," Trends in Neurosciences, vol. 32, no. 12, pp. 638-647, 2009.

[24] M. V. Sofroniew and H. V. Vinters, "Astrocytes: biology and pathology," Acta Neuropathologica, vol. 119, no. 1, pp. 7-35, 2010.

[25] J. De Keyser, G. Laureys, F. Demol, N. Wilczak, J. Mostert, and R. Clinckers, "Astrocytes as potential targets to suppress inflammatory demyelinating lesions in multiple sclerosis," Neurochemistry International, vol. 57, no. 4, pp. 446-450, 2010.

[26] C. S. Moore, S. L. Abdullah, A. Brown, A. Arulpragasam, and S. J. Crocker, "How factors secreted from astrocytes impact myelin repair," Journal of Neuroscience Research, vol. 89, no. 1, pp. 13-21, 2011.

[27] A. Williams, G. Piaton, and C. Lubetzki, "Astrocytes-friends or foes in multiple sclerosis?” Glia, vol. 55, no. 13, pp. 1300-1312, 2007.

[28] R. P. Bunge, W. R. Puckett, J. L. Becerra, A. Marcillo, and R. M. Quencer, "Observations on the pathology of human spinal cord injury. A review and classification of 22 new cases with details from a case of chronic cord compression with extensive focal demyelination," Advances in Neurology, vol. 59, pp. 75-89, 1993.

[29] A. M. Butt and M. Berry, "Oligodendrocytes and the control of myelination in vivo: new insights from the rat anterior medullary velum," Journal of Neuroscience Research, vol. 59, no. 4, pp. 477-488, 2000.

[30] E. M. Frohman, M. K. Racke, and C. S. Raine, "Medical progress: multiple sclerosis-the plaque and its pathogenesis," The New England Journal of Medicine, vol. 354, no. 9, pp. 942-955, 2006.

[31] J. E. Holley, D. Gveric, J. Newcombe, M. L. Cuzner, and N. J. Gutowski, "Astrocyte characterization in the multiple sclerosis glial scar," Neuropathology and Applied Neurobiology, vol. 29, no. 5, pp. 434-444, 2003.

[32] H. S. Keirstead, G. Nistor, G. Bernal et al., "Human embryonic stem cell-derived oligodendrocyte progenitor cell transplants remyelinate and restore locomotion after spinal cord injury," Journal of Neuroscience, vol. 25, no. 19, pp. 4694-4705, 2005.

[33] R. C. Sergott, M. J. Brown, and R. M.-D. Polenta, "Optic nerve demyelination induced by human serum: patients with multiple sclerosis or optic neuritis and normal subjects," Neurology, vol. 35, no. 10, pp. 1438-1442, 1985.

[34] W. T. Norton, D. A. Aquino, I. Hozumi, F.-C. Chiu, and C. F. Brosnan, "Quantitative aspects of reactive gliosis: a review," Neurochemical Research, vol. 17, no. 9, pp. 877-885, 1992.

[35] J. G. M. C. Damoisequx, E. A. Dopp, W. Calame, D. Chao, G. G. MacPherson, and C. D. Dijkstra, "Rat macrophage lysosomal membrane antigen recognized by monoclonal antibody ED1," Immunology, vol. 83, no. 1, pp. 140-147, 1994.
[36] C. D. Dijkstra, E. A. Dopp, P. Joling, and G. Kraal, "The heterogeneity of mononuclear phagocytes in lymphoid organs: distinct macrophage subpopulations in the rat recognized by monoclonal antibodies ED1, ED2 and ED3," Immunology, vol. 54, no. 3, pp. 589-599, 1985.

[37] C. D. Dijkstra, E. A. Döpp, T. K. Van Den Berg, and J. G. M. C. Damoiseaux, "Monoclonal antibodies against rat macrophages," Journal of Immunological Methods, vol. 174, no. 1-2, pp. 21-23, 1994.

[38] J. K. Dyer, J. A. Bourque, and J. D. Steeves, “The role of complement in immunological demyelination of the mammalian spinal cord," Spinal Cord, vol. 43, no. 7, pp. 417-425, 2005.

[39] S. L. Carlson, M. E. Parrish, J. E. Springer, K. Doty, and L. Dossett, "Acute inflammatory response in spinal cord following impact injury," Experimental Neurology, vol. 151, no. 1, pp. 77-88, 1998.

[40] I. Dusart and M. E. Schwab, "Secondary cell death and the inflammatory reaction after dorsal hemisection of the rat spinal cord," European Journal of Neuroscience, vol. 6, no. 5, pp. 712724, 1994.

[41] P. G. Popovich, P. Wei, and B. T. Stokes, "Cellular inflammatory response after spinal cord injury in Sprague-Dawley and Lewis rats," Journal of Comparative Neurology, vol. 377, no. 3, pp. 443464, 1997.

[42] Z. Zhang, C. J. Krebs, and L. Guth, "Experimental analysis of progressive necrosis after spinal cord trauma in the rat: etiological role of the inflammatory response," Experimental Neurology, vol. 143, no. 1, pp. 141-152, 1997.

[43] M. T. Fitch, C. Doller, C. K. Combs, G. E. Landreth, and J. Silver, "Cellular and molecular mechanisms of glial scarring and progressive cavitation: in vivo and in vitro analysis of inflammation-induced secondary injury after CNS trauma," Journal of Neuroscience, vol. 19, no. 19, pp. 8182-8198, 1999.

[44] M. T. Fitch and J. Silver, "Activated macrophages and the bloodbrain barrier: inflammation after CNS injury leads to increases in putative inhibitory molecules," Experimental Neurology, vol. 148, no. 2, pp. 587-603, 1997.

[45] S. J. Hill-Felberg, T. K. McIntosh, D. L. Oliver, R. Raghupathi, and E. Barbarese, "Concurrent loss and proliferation of astrocytes following lateral fluid percussion brain injury in the adult rat," Journal of Neuroscience Research, vol. 57, no. 2, pp. 271-279, 1999.

[46] E. Preston, J. Webster, and D. Small, "Characteristics of sustained blood-brain barrier opening and tissue injury in a model for focal trauma in the rat," Journal of Neurotrauma, vol. 18, no. 1, pp. 83-92, 2001.

[47] W. F. Blakemore, D. M. Chari, J. M. Gilson, and A. J. Crang, "Modelling large areas of demyelination in the rat reveals the potential and possible limitations of transplanted glial cells for remyelination in the CNS," Glia, vol. 38, no. 2, pp. 155-168, 2002.

[48] R. Reynolds and G. P. Wilkin, "Cellular reaction to an acute demyelinating/remyelinating lesion of the rat brain stem: localisation of G(D3) ganglioside immunoreactivity," Journal of Neuroscience Research, vol. 36, no. 4, pp. 405-422, 1993.

[49] R. H. Woodruff and R. J. Franklin, "Demyelination and remyelination of the caudal cerebellar peduncle of adult rats following stereotaxic injections of lysolecithin, ethidium bromide, and complement/anti-galactocerebroside: a comparative study," Glia, vol. 25, no. 3, pp. 216-228, 1999.

[50] F. L. Mastaglia, W. M. Carroll, and A. R. Jennings, "Spinal cord lesions induced by antigalactocerebroside serum," Clinical and Experimental Neurology, vol. 26, pp. 33-44, 1989. 
[51] H. Aldskogius, "Repairing CNS myelin-astrocytes have to do their jobs," Experimental Neurology, vol. 192, no. 1, pp. 7-10, 2005.

[52] W. F. Blakemore, J. M. Gilson, and A. J. Crang, "The presence of astrocytes in areas of demyelination influences remyelination following transplantation of oligodendrocyte progenitors," Experimental Neurology, vol. 184, no. 2, pp. 955-963, 2003.

[53] R. J. Franklin, A. J. Crang, and W. F. Blakemore, "The role of astrocytes in the remyelination of glia-free areas of demyelination," Advances in Neurology, vol. 59, pp. 125-133, 1993.

[54] A. Meyer-Franke, S. Shen, and B. A. Barres, "Astrocytes induce oligodendrocyte processes to align with and adhere to axons," Molecular and Cellular Neurosciences, vol. 14, no. 4-5, pp. 385397, 1999.

[55] J. F. Talbott, D. N. Loy, Y. Liu et al., "Endogenous Nkx2. ${ }^{2+} / \mathrm{Olig}^{2+}$ oligodendrocyte precursor cells fail to remyelinate the demyelinated adult rat spinal cord in the absence of astrocytes," Experimental Neurology, vol. 192, no. 1, pp. 11-24, 2005.

[56] S. Elsworth and J. M. Howell, "Variation in the response of mice to cuprizone," Research in Veterinary Science, vol. 14, no. 3, pp. 385-387, 1973.

[57] M. M. Hiremath, Y. Saito, G. W. Knapp, J. P.-Y. Ting, K. Suzuki, and G. K. Matsushima, "Microglial/macrophage accumulation during cuprizone-induced demyelination in C57BL/6 mice," Journal of Neuroimmunology, vol. 92, no. 1-2, pp. 38-49, 1998.

[58] T. Skripuletz, D. Hackstette, K. Bauer et al., "Astrocytes regulate myelin clearance through recruitment of microglia during cuprizone-induced demyelination," Brain, vol. 136, part 1, pp. 147-167, 2013.

[59] M. L. Fuller, A. K. DeChant, B. Rothstein et al., "Bone morphogenetic proteins promote gliosis in demyelinating spinal cord lesions," Annals of Neurology, vol. 62, no. 3, pp. 288-300, 2007.

[60] A. Gadea, S. Schinelli, and V. Gallo, "Endothelin-1 regulates astrocyte proliferation and reactive gliosis via a JNK/c-Jun signaling pathway," Journal of Neuroscience, vol. 28, no. 10, pp. 2394-2408, 2008.

[61] G. L. Hinks and R. J. M. Franklin, "Distinctive patterns of PDGF-A, FGF-2, IGF-I, and TGF- $\beta 1$ gene expression during remyelination of experimentally-induced spinal cord demyelination," Molecular and Cellular Neurosciences, vol. 14, no. 2, pp. 153-168, 1999.

[62] C. A. Dyer and J. A. Benjamins, "Antibody to galactocerebroside alters organization of oligodendroglial membrane sheets in culture," Journal of Neuroscience, vol. 8, no. 11, pp. 4307-4318, 1988.

[63] J. Bauer, F. Berkenbosch, A.-M. Van Dam, and C. D. Dijkstra, "Demonstration of interleukin- $\beta$ in Lewis rat brain during experimental allergic encephalomyelitis by immunocytochemistry at the light and ultrastructural level," Journal of Neuroimmunology, vol. 48, no. 1, pp. 13-22, 1993.

[64] D. Giulian and L. B. Lachman, "Interleukin-1 stimulation of astroglial proliferation after brain injury," Science, vol. 228, no. 4698, pp. 497-499, 1985.

[65] L. M. Herx, S. Rivest, and V. W. Yong, "Central nervous systeminitiated inflammation and neurotrophism in trauma: IL- $1 \beta$ is required for the production of ciliary neurotrophic factor," Journal of Immunology, vol. 165, no. 4, pp. 2232-2239, 2000.

[66] L. M. Herx and V. W. Yong, "Interleukin- $1 \beta$ is required for the early evolution of reactive astrogliosis following CNS lesion," Journal of Neuropathology and Experimental Neurology, vol. 60, no. 10, pp. 961-971, 2001.
[67] M. Rostworowski, V. Balasingam, S. Chabot, T. Owens, and V. W. Yong, "Astrogliosis in the neonatal and adult murine brain post-trauma: elevation of inflammatory cytokines and the lack of requirement for endogenous interferon- $\gamma$," Journal of Neuroscience, vol. 17, no. 10, pp. 3664-3674, 1997.

[68] T. R. Sairanen, P. J. Lindsberg, M. Brenner, A.-L. Sirén, and T. R. Vidman, "Global forebrain ischemia results in differential cellular expression of interleukin- $1 \beta$ (IL-1 $\beta$ ) and its receptor at mRNA and protein level," Journal of Cerebral Blood Flow and Metabolism, vol. 17, no. 10, pp. 1107-1120, 1997.

[69] C.-S. Chiang, A. Stalder, A. Samimi, and I. L. Campbell, "Reactive gliosis as a consequence of interleukin-6 expression in the brain: studies in transgenic mice," Developmental Neuroscience, vol. 16, no. 3-4, pp. 212-221, 1994.

[70] M. A. Klein, J. C. Moller, L. L. Jones, H. Bluethmann, G. W. Kreutzberg, and G. Raivich, "Impaired neuroglial activation in interleukin-6 deficient mice," Glia, vol. 19, no. 3, pp. 227-233, 1997.

[71] S. Okada, M. Nakamura, Y. Mikami et al., "Blockade of interleukin-6 receptor suppresses reactive astrogliosis and ameliorates functional recovery in experimental spinal cord injury," Journal of Neuroscience Research, vol. 76, no. 2, pp. 265-276, 2004.

[72] C. Bogdan, Y. Vodovotz, and C. Nathan, "Macrophage deactivation by interleukin 10," Journal of Experimental Medicine, vol. 174, no. 6, pp. 1549-1555, 1991.

[73] A. D’Andrea, M. Aste-Amezaga, N. M. Valiante, X. Ma, M. Kubin, and G. Trinchieri, "Interleukin 10 (IL-10) inhibits human lymphocyte interferon $\gamma$-production by suppressing natural killer cell stimulatory factor/IL-12 synthesis in accessory cells," Journal of Experimental Medicine, vol. 178, no. 3, pp. 1041-1048, 1993.

[74] R. De Waal Malefyt, J. Abrams, B. Bennett, C. G. Figdor, and J. E. De Vries, "Interleukin 10(IL-10) inhibits cytokine synthesis by human monocytes: an autoregulatory role of IL-10 produced by monocytes," Journal of Experimental Medicine, vol. 174, no. 5, pp. 1209-1220, 1991.

[75] D. F. Fiorentino, A. Zlotnik, T. R. Mosmann, M. Howard, and A. O'Garra, "IL-10 inhibits cytokine production by activated macrophages," Journal of Immunology, vol. 147, no. 11, pp. 38153822, 1991.

[76] T. R. Mosmann, "Properties and functions of interleukin-10," Advances in Immunology, vol. 56, pp. 1-26, 1994.

[77] V. Balasingam and V. W. Yong, "Attenuation of astroglial reactivity by interleukin-10," Journal of Neuroscience, vol. 16, no. 9, pp. 2945-2955, 1996.

[78] T. Abo, C. A. Miller, G. L. Gartland, and C. M. Balch, "Differentiation stages of human natural killer cells in lymphoid tissues from fetal to adult life," Journal of Experimental Medicine, vol. 157, no. 1, pp. 273-284, 1983.

[79] P. De Paoli, S. Battistin, and G. F. Santini, "Age-related changes in human lymphocyte subsets: progressive reduction of the CD4 CD45R (suppressor inducer) population," Clinical Immunology and Immunopathology, vol. 48, no. 3, pp. 290-296, 1988.

[80] I. Hannet, F. Erkeller-Yuksel, P. Lydyard, V. Deneys, and M. DeBruyere, "Developmental and maturational changes in human blood lymphocyte subpopulations," Immunology Today, vol. 13, no. 6, pp. 215-218, 1992.

[81] C. Y. Lu, "The delayed ontogenesis of Ia-positive macrophages: implications for host defense and self-tolerance in the neonate," 
Clinical and Investigative Medicine, vol. 7, no. 4, pp. 263-267, 1984.

[82] V. Balasingam, T. Tejada-Berges, E. Wright, R. Bouckova, and V. W. Yong, "Reactive astrogliosis in the neonatal mouse brain and its modulation by cytokines," Journal of Neuroscience, vol. 14, no. 2, pp. 846-856, 1994.

[83] S. S. Ousman and S. David, "MIP-1 $\alpha$, MCP-1, GM-CSF, and TNF- $\alpha$ control the immune cell response that mediates rapid phagocytosis of myelin from the adult mouse spinal cord," Journal of Neuroscience, vol. 21, no. 13, pp. 4649-4656, 2001.

[84] C. Zhao, W.-W. Li, and R. J. M. Franklin, "Differences in the early inflammatory responses to toxin-induced demyelination are associated with the age-related decline in CNS remyelination," Neurobiology of Aging, vol. 27, no. 9, pp. 1298-1307, 2006.

[85] C. Schachtrup, J. K. Ryu, M. J. Helmrick et al., "Fibrinogen triggers astrocyte scar formation by promoting the availability of active TGF- $\beta$ after vascular damage," Journal of Neuroscience, vol. 30, no. 17, pp. 5843-5854, 2010.

[86] K. Beck and C. Schachtrup, "Vascular damage in the central nervous system: a multifaceted role for vascular-derived TGFß," Cell and Tissue Research, vol. 347, no. 1, pp. 187-201, 2012. 


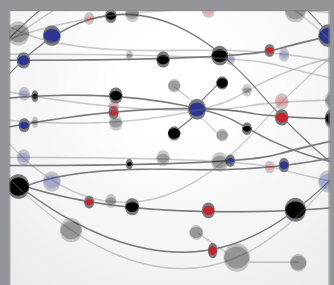

The Scientific World Journal
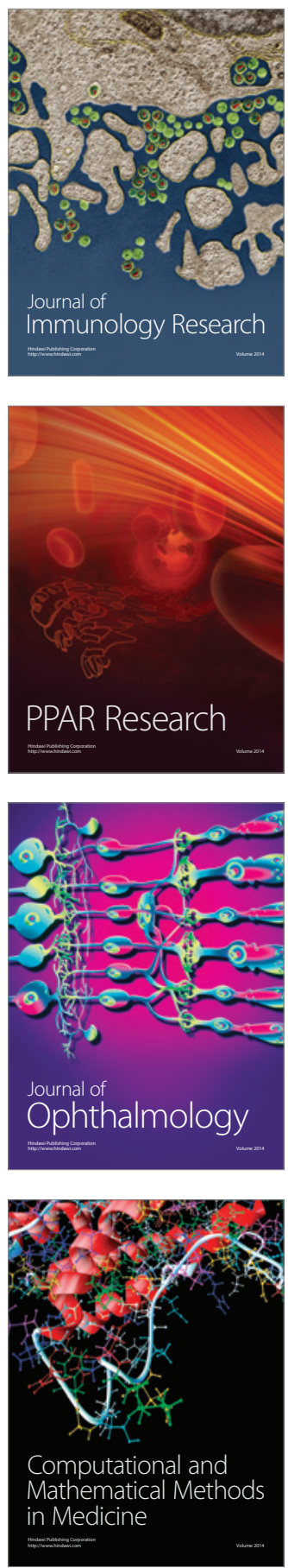

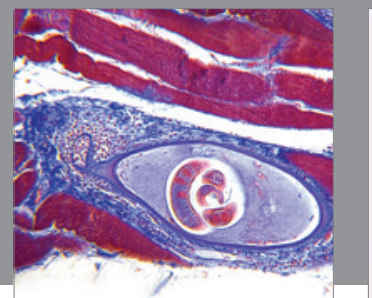

Gastroenterology

Research and Practice
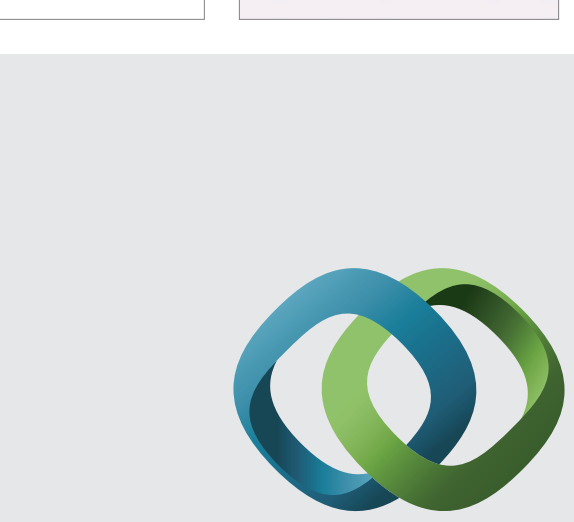

\section{Hindawi}

Submit your manuscripts at

http://www.hindawi.com
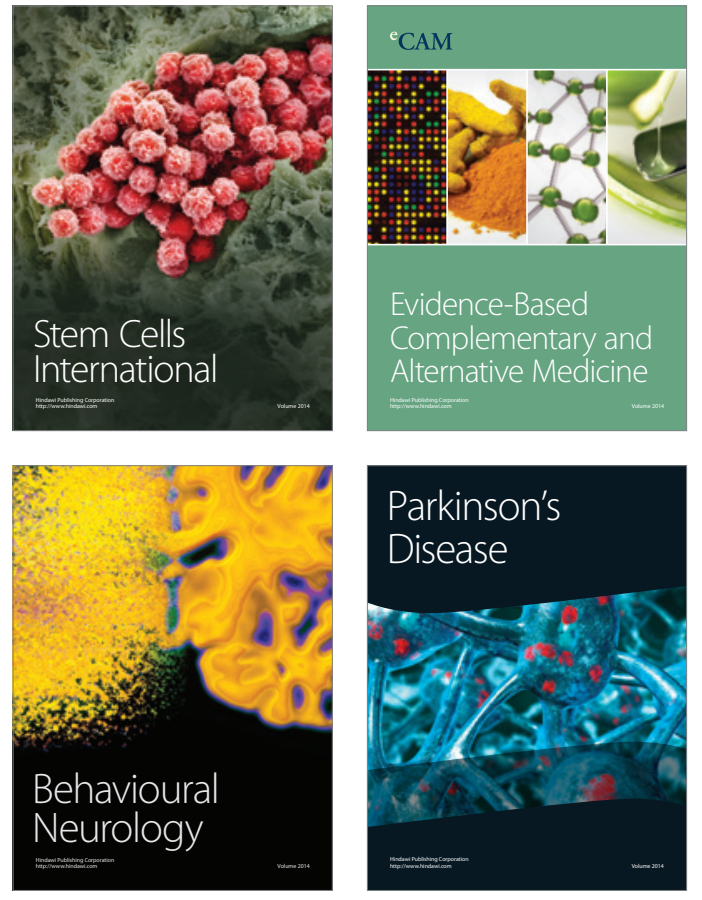
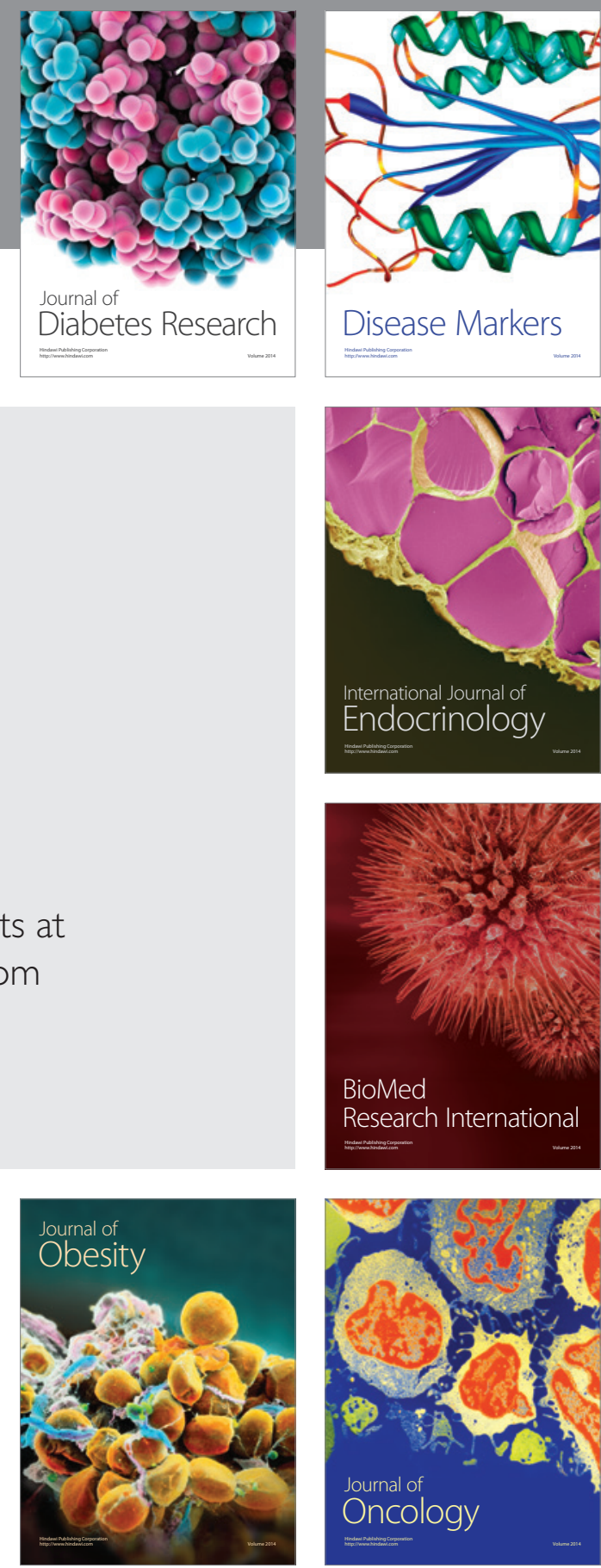

Disease Markers
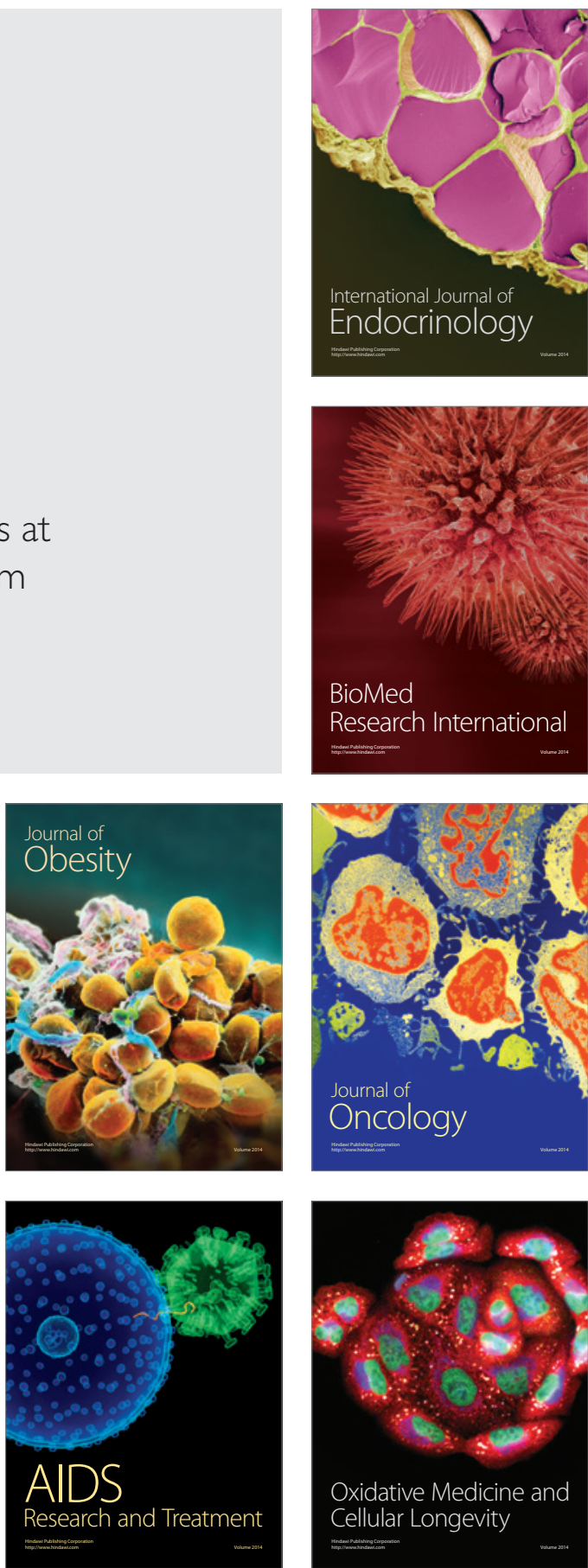